\title{
CONDITIONS IMPLYING CONTINUITY OF OPEN AND CLOSED FUNCTIONS
}

\author{
IVAN BAGGS
}

\begin{abstract}
A.BSTRACT. It is shown that every open and closed real valued function of a real variable is continuous. This result is then extended to the case where the domain space is first countable and regular for a special class of open and closed functions.
\end{abstract}

1. Introduction. Let $X$ and $Y$ be topological spaces and let $R$ denote the real iine with the usual topology. A function $f: X \rightarrow Y$ is open (closed) if for every open (closed) subset $U$ of $X, f(U)$ is open (closed) in $Y$.

It is well known that an open (closed) function $f: X \rightarrow Y$ may not be continuous. This still holds when $X=Y=R$. It was shown by $\mathrm{S}$. Marcus (see [5]) that an open real valued function of a real variable may be everywhere discontinuous. In fact, by modifying Example 6.1 of [1], it follows that given any nonvoid set $A \subseteq R$, there exists a function $f: R \rightarrow R$ such that $f(B)=A$, for every uncountable Borel set, $B$. As was pointed out to me by the referee, by taking $A=R$ in this example, we have a function which maps every open set onto $R$ and takes every uncountable closed set to a closed set. Yet, this function is everywhere discontinuous.

The purpose of this paper is to show that functions which are open and, in addition, take countable closed sets to closed sets are much better behaved. It will follow from Theorem 3 that every open and closed function from $R$ into $R$ is homeomorphism. Theorem 4 and Theorem 5 will extend this result to more general spaces.

If $G$ is a set, $\operatorname{cl} G$ will denote the closure of $G, F(G)$ will denote the boundary of $G$ and $G^{c}$ will denote the complement of $G$. The notation $x_{n} \rightarrow x$ is used for a sequence $\left\{x_{n}\right\}$ converging to $x$. A regular space is also assumed to be Hausdorff.

Part of this paper is an extract from the author's doctoral dissertation written at the University of Alberta under the supervision of Professor S. Willard. The suggestions of Professor S. Willard, A. Wilansky and the referee are gratefully acknowledged.

Received by the editors December 3, 1970.

AMS 1970 subject classifications. Primary 54C10; Secondary 26A15.

Key words and phrases. Open functions, closed functions, peripherally continuous functions, continuous functions, first countable regular spaces.

c. American Msthematical Society 1972 
2. Open and closed functions. Our main aim in this section is to show that every open and closed real valued function of a real variable is continuous (Theorem 3). In proving this and the other theorems of the sequel involving closed functions, we really only need functions which take countable closed sets to closed sets. However, since all spaces are assumed to be first countable, it follows from Lemma 1 that there is no loss in generality by assuming that $f$ is closed.

Lemma 1. Let $X$ and $Y$ be first countable Hausdorff spaces. A function $f: X \rightarrow Y$ is closed if and only if it maps countable closed sets to closed sets.

Proof. Suppose $f$ maps countable closed sets to closed sets. Assume $K$ is a closed subset of $X$ such that $f(K)$ is not closed. Let $y$ be a limit point of $f(K)$ such that $y \notin f(K)$. For each positive integer $n$, choose $y_{n} \in$ $f(K)$ such that $y_{n} \rightarrow y$. Let $x_{n} \in f^{-1}\left(y_{n}\right) \cap K$, for $n=1,2, \cdots$. Then $P=$ $\left\{x_{n} \mid n=1,2, \cdots\right\}$ is not a closed subset of $X$. Let $x \in K$ be such that $x \notin P$ and $x$ is a limit point of $P$. Let $Q=\left\{x_{i} \mid i=1,2, \cdots\right\}$ be a subsequence of $P$ such that $x_{i} \rightarrow x$. Then $Q \cup\{x\}$ is a countable closed subset of $X$ such that $f(Q \cup\{x\})$ is not a closed subset of $Y$. A contradiction.

Since the converse is obvious, the lemma is established.

LEMMA 2. Let $f: X \rightarrow Y$ be a closed function, where $X$ and $Y$ are first countable Hausdorff spaces and $Y$ has at least two limit points. If $x$ is a limit point of $X$, then there exisis some neighbourhood $U$ of $x$ such that $f(U)$ is a proper subset of $Y$.

Proof. Let $U_{n}, n=1,2, \cdots$, be a decreasing sequence of open sets which form a local neighbourhood base at $x$. Suppose for each $n, f\left(U_{n}\right)=Y$. Let $y$ be a limit point of $Y$ such that $y \neq f(x)$ and let $y_{n}, n=1,2, \cdots$, be a sequence of distinct points in $Y$ such that $y_{n} \rightarrow y$. Let $x_{n} \in f^{-1}\left(y_{n}\right) \cap U_{n}$, for $n=1,2, \cdots$. Then $x_{n} \rightarrow x$ and so $\left\{x_{n} \mid n=1,2, \cdots\right\} \cup\{x\}$ is a closed subset of $X$. However, $f\left(\left\{x_{n} \mid n=1,2, \cdots\right\} \cup\{x\}\right)$ is not a closed set in $Y$, which contradicts the hypothesis that $f$ is a closed function. Hence, for some $n$, $f\left(U_{n}\right)$ must be a proper subset of $Y$.

COROLlaRY 1. Let $f: X \rightarrow R$ be a closed function, where $X$ is a first countable Hausdorff space. Let $x$ be a limit point of $X$. If $V$ is any neighbourhood of $f(x)$, then there exists a neighbourhood $U$ of $x$ such that $V$ is not a subset of $f(U)$.

Proof. Suppose for every neighbourhood $U$ of $x, f(U) \supset V$. Let $y$ be a limit point of $V$ such that $y \neq f(x)$. Now, as in the proof of Lemma 2 , construct sequences $y_{n} \rightarrow y$ and $x_{n} \rightarrow x$ such that $f\left(x_{n}\right)=y_{n}$. Then $f\left(\left\{x_{n} \mid n=1,2, \cdots\right\} \cup\{x\}\right)$ is not a closed set, which contradicts the fact that $f$ is a closed function. 
REMARK 1. It was pointed out earlier that there exists a function $f: R \rightarrow R$ such that $f$ maps every open set and every uncountable closed set onto $R$. It follows from the preceding corollary that $f$ cannot be closed. Also, if $x \in R$ and $f$ is a closed function from $R$ into $R$, then there exists some neighbourhood $U$ of $x$ such that $f(U)$ does not contain an unbounded interval containing $f(x)$. However, if $x_{0}$ is any fixed element of $R$, then it is possible to construct a closed function $f: R \rightarrow R$ such that for every neighbourhood $U$ of $x_{0} . f(U)$ contains an unbounded interval. For example, put $f(x)=x$, if $x \notin\left(x_{0}, x_{0}+1\right)$; and define $f$ on $\left(x_{0}, x_{0}+1\right]$ such that $f$ maps $\left(x_{0}, x_{0}+1\right]$ homeomorphically onto $\left[x_{\theta}+1,+\infty\right)$.

THEOREM 1. If $f: R \rightarrow R$ is both open and closed, then for each $x \in R$ there exists a closed neighbourhood $K$ of $x$ such that $f(K)$ is compact.

Proof. Let $x$ be a fixed element of $R$ and denote by $U_{n}$ the open interval $\left(a_{n}, b_{n}\right)$, where $a_{n}=x-1 / n$ and $b_{n}=x+1 / n$, for $n=1,2, \cdots$.

We claim $f\left(\mathrm{cl} U_{n}\right)$ is compact for some $n$. Suppose not. Since $f$ is a closed function, $f\left(\mathrm{cl} U_{n}\right)$ is a closed subset of $R$. Hence, $f\left(\mathrm{cl} U_{n}\right)$ must be unbounded for each $n$. But by Corollary 1, there is scme positive integer $N$ such that $f\left(\mathrm{cl} l_{N}\right)$ does not contain an unbounded interval containing $f(x)$. If follows that $f\left(\mathrm{cl} U_{N}\right)$, and $f\left(U_{N}\right)$ as well, is disconnected. Now, $f$ is an open function, so $f\left(U_{N}\right)$ can be written as the union of a sequence $G_{1}, G_{2}, G_{3}, \cdots$, of at least two, disjoint, nonempty, open intervals. If $f(x) \in G_{1}$, say, then $G_{1}$ is bounded since $f\left(U_{N}\right)$ contains no unbounded interval containing $f(x)$.

But now $f\left(U_{N}\right)=G_{1} \cup\left\{\bigcup_{k=1} G_{k}\right\}$ has at least three distinct limit points, $e_{1}, e_{2}$ and $e_{3}$ (the endpoints of the bounded interval $G_{1}$ and at least one other endpoini of at least one more open interval; these points must be distinct since $f\left(\mathrm{cl} U_{N}\right)$ is disconnected) which do not belong to $f\left(U_{N}\right)$. But, since we assumed that $U_{N}$ is the open interval $\left(a_{N}, b_{N}\right)$, this shows that $f\left(\mathrm{cl} U_{N}\right)=f\left(U_{N}\right) \cup\left\{f\left(a_{N}\right)\right\} \cup\left\{f\left(b_{N}\right)\right\}$ cannot be a closed subset of $R$. A contradiction. So, for some $n, f\left(\mathrm{cl} U_{n}\right)$ is compact and the theorem is established.

In order to establish the main theorem, we need the following result by Klee and Utz:

Theorem 2 [4, Theorem B]. Let $X$ and $Y$ be metric spaces. If $X$ is locally connected at $x$ and $f: X \rightarrow Y$ takes compact sets to compact sets and connected sets to connected sets, then $f$ is continuous at $x$.

THEOREM 3. Let $f$ be an open and closed function from $R$ into $R$, then $f$ is continuous. 
Proof. Let $x \in R$. By Theorem 1, there exists a closed neighbourhood $K$ of $x$ such that $f(K)$ is compact. Let $I=(a, b)$ be an open interval in $R$ such that $I \subset K$. If $f(I)$ is disconnected, then, since $f(I)$ is open and bounded, it must have at least three distinct limit points $u_{1}, u_{2}$ and $u_{3}$, such that $u_{i} \notin f(I)$, for $i=1,2,3$. However, this is impossible, since $f(\mathrm{cl} I)=$ $f(I) \cup\{f(a)\} \cup\{f(b)\}$ is closed in $R$. Therefore, $f(I)$ is open and connected. Since $f(\mathrm{cl} I)$ is closed, $f(\mathrm{cl} I)$ must also be connected. Therefore, the restriction of $f$ to $K$ takes compact sets to compact sets and connected sets to connected sets. It follow's from Theorem 2 , that the restriction of $f$ to $K$ is continuous, and since $x$ is an interior point of $K, f$ is continuous at $x$.

COROLlARY 2. Every open and closed function from $R$ inio $R$ is a homeomorphism.

Proof. It follows from the preceding theorem that if $f: R \rightarrow R$ is open and closed, then it is continuous. It is easily shown that an open and continuous function from $R$ into $R$ is morotone. Hence, $f$ is a homeomorphism.

It is well known [2, p. 227] that if $X$ is a compact Hausdorff space, then $p$, the projection map from $X \times Y$ into $Y$, is open, closed and continuous. So, for more general space, Coroliary 2 does not hold even when $f$ is continuous. We now give an example of an open and closed function $f$, from a closed subset $T$ of the plane, into $R$, such that $f$ is not continuous on $T$.

EXAMPLE 1. Let $T$ be the subset of the plane consisting of the $X$-axis and the positive $Y$-axis. Let the topology on $T$ be that inherited from the plane. Define $f: T \rightarrow R$ as follows:

If $p \in T$ and $p=(x, 0)$, then put $f(p)=x$; define $f$ on the $Y$-axis so that the positive $Y$-axis is mapped homeomorphically cnto $R$. If $V$ is any open (closed) subset of $T$, it is immediate that $f(V)$ is open (closed). However, $f$ is not continuous at $p \in T$, if $p=(0,0)$.

3. Open and closed functions on first countable regular spaces. A function $f$ from $X$ into $Y$ is peripherally continuous [3] if and only if for each $x \in X$ and for each neighbourhood $U$ and $V$ of $x$ and $f(x)$, respectively, there exists an open set $W$ such that $x \in W^{\prime} \subset U$ and $f(F(W))=V$. The function constructed in Example 1 is not peripherally continuous.

THEOREM 4. Let $X$ be a first countable and regular topologicai space. An open and closed function $f$ from $X$ into $R$ is continuous if and only if it is peripherally continuous.

Proof. It is clear that if $f$ is continuous then $f$ is peripherally continuous. Conversely, let $f$ be peripherally continuous. First, we will show that for each $x \in X$, there exists some closed neighbourhood $U$ of $x$ such that $f(U)$ 
is compact. Let $U_{n}, n=1,2,3, \cdots$, be a decreasing sequence of open sets which form a local base at $x$, such that for each $n, U_{n} \supset \operatorname{cl} U_{n+1}$. Suppose for each $n, f\left(\mathrm{cl} U_{n}\right)$ is not compact. Then, since $f$ is a closed function, $f\left(\mathrm{cl} U_{n}\right)$ is unbounded, for $n=1,2, \cdots$. Hence, by Corollary 1 , there must exist some positive integer $N$ such that $f\left(U_{N}\right)$ does not contain an unbounded interval containing $f(x)$. Therefore, $f\left(\mathrm{cl} U_{N}\right)$ is disconnected and so $f^{\prime}$ (cl $\left.U_{v}\right) \subset A_{1} \cup A_{2}$, where $A_{1}$ and $A_{2}$ are two disjoint open intervals in $R$, and $f\left(\mathrm{cl} U_{N}\right) \cap A_{1}$ and $f\left(\mathrm{cl} U_{N}\right) \cap A_{2}$ are nonempty subsets of $R$. We may assume that $f(x) \in A_{1}$ and $A_{1}$ is bounded. Further, if $U$ is any neighbourhood of $x$, then $f(U) \cap A_{1}$ and $f\left(U \cap A_{\Omega}\right)$ are both nonempty sets since $f\left(U_{n}\right)$ is unbounded for $n=1,2, \cdots$. But. $f$ is peripherally continuous so there exists an open set $G$ such that $x \in G \subset U_{Y}$ ard $f(F(G)) \subset A_{1}$. However, $f(\mathrm{cl} G)=f(G) \cup f(F(G))$ is closed in $R$, so since $f(G) \cap A_{2}$ is a nonempty open set, there must exist $y \in F(G)$ such that $f(y) \in A_{2}$. This, however, would contradict the fact that $f(F(G)) \subset A_{1}$. Hence, there exists some $n$, such that $f\left(\right.$ ci $\left.U_{n}\right)$ is compact.

If $f$ is not continuous at $x$, then there exists some neighbourhood $V$ of $f(x)$ such that for every neighbourhood $G$ of $x, f(G) \nsubseteq V$. So, since $f\left(U_{n}\right)$ is open in $R$, there exists an uncountable set $K_{n} \subset U_{n}$ such that $f\left(K_{n}\right) \subset V^{c}$, for $n=1,2, \cdots$. Let $N$ be a positive integer such that $f\left(\operatorname{cl} U_{n}\right)$ is compact, for all $n \geqq N$. Let $x_{i} \in U_{i}$, for $i \geqq N$, such that $f\left(x_{i}\right) \in V^{c}$ and if $i \neq j$, then $f\left(x_{i}\right) \neq f\left(x_{j}\right) . f\left(\mathrm{cl} U_{n}\right)$ is compact for every $n \geqq N$, therefore, the sequence $f\left(x_{n}\right), n=N, N+1, \cdots$, has a subsequence $f\left(x_{k}\right), k=1,2, \cdots$, such that $f\left(x_{k}\right) \rightarrow p \in f\left(\mathrm{cl} U_{N}\right)$. Further, we can assume that $f\left(x_{k}\right), k=1,2, \cdots$, has been chosen such that $p \neq f\left(x_{k}\right)$, for $k=1,2, \cdots$. But this implies that $\left\{x_{k} \mid k=1,2, \cdots\right\} \cup\{x\}$ is a closed set in $X$ whose image is not closed in $R$. A contradiction, so $f$ is continuous at $x$.

THEOREM 5. Let $X$ and $Y$ be first countable Hausdorff spaces. If $Y$ is also compact and perfect, then every open and closed function from $X$ into $Y$ is continuous.

Proof. Let $f: X \rightarrow Y$ be an open and closed function. Suppose there exists some $x \in X$ such that $f$ is not continuous at $x$. Let $U_{n}, n=1,2, \cdots$, be a decreasing sequence of open sets which form a local neighbourhood base at $x$. Since $f$ is discontinuous at $x$, and $X$ is regular, there exists a closed neighbourhood $V$ of $f(x)$ such that $f\left(U_{n}\right) \cap V^{c}$ is nonempty, for $n=1,2, \cdots$. Put $V_{n}=f\left(U_{n}\right)-V$, for $n=1,2, \cdots$. By assumption $f$ is open and $Y$ has no isolated point, so $V_{n}$ is open and infinite for each $n$. For $n=1,2, \cdots$, let $x_{n} \in U_{n}$ such that $f\left(x_{n}\right) \in V_{n}$ and if $i \neq j$, then $f\left(x_{i}\right) \neq$ $f\left(x_{j}\right)$. Now, since $K=\mathrm{cl}\left(\bigcup_{n} V_{n}\right)$ is compact. there must exist a subsequence $\left\{x_{k}\right\}, k=1,2, \cdots$, of $\left\{x_{n}\right\}, n=1,2, \cdots$, such that $f\left(x_{k}\right) \rightarrow p \in K$. We may assume, since $f\left(x_{i}\right) \neq f(x$,$) , for i \neq j$, that the subsequence $x_{k}$, 
$k=1,2, \cdots$, has been chosen such that $f\left(x_{k}\right) \neq p$, for $k=1,2, \cdots$. This implies that $f\left(\left\{x_{k} \mid k=1,2, \cdots\right\} \cup\left\{x_{i}\right)\right.$ is not a closed set. A contradiction. Therefore $f$ is continuols at $x$ and the theorem is established.

It follows from Example 1, that the compactness condition on $Y$ cannot be omitted in the preceding theorem. Also we cannot drop the requirement that $X$ is Hausdorff. For, if $(Y, \tau)$ is a compact Hausdorff space and $\sigma$ is any strictly smaller topology on $Y$, then $(Y, \sigma)$ is not Hausdorff and the identity mapping from $(Y, \sigma)$ onto $(Y, \tau)$ is open and closed but not continuous. The next example shows that Theorem 5 is false if $Y$ is not perfect.

EXAMPlÉ 2. Put $Y=\{0,1\}$ with the discrete topology. Let $X=$ $\{(1 / n) \mid n=1,2, \cdots\} \cup\{x\}$ and let the topology on $X$ be that inherited from $R$. Put $f(1 / n)=0$, if $n$ is even, and $f(x)=1$, otherwise, for $x \in X$. Then $f$ is an open and closed function from $X$ onto $Y$ but $f$ is not continuous.

REMARK 2. It is unknown whether we can remove either first countability from Theorems 4 and 5 or regularity from Theorem 4.

\section{REFERENCES}

1. A. M. Bruckner and J. G. Ceder, Darboux continuity, Jber. Deutsch Math.Verein. 67 (1964/65), Abt. 1, 93-117. MR $32 \# 421 \%$.

2. J. Dugundji, Topoligy, Allyn and Bacon, Boston, Mass., 1966. MR 33 \#1824.

3. O. H. Hamilton, Fixed points for certuin noncontinuous transformations, Proc. Amer. Math. Soc. 8 (1957), 750-756. MR 19. 301.

4. V. L. Klee, Jr., and W. R. Utz, Some remarks on continuoits transformations, Proc. Amer. Math. Soc. 5 (1954), 182-184. MR 15. 730.

5. S. Marcus, Open everywhere discontinuous functions, Amer. Math. Monthly 72 (1965), 993-995. MR 32 \#2528.

Department of Mathematic's, St. Francis Xavier University, Antigonish, Nova Scotia, Canada 